Nonlin. Processes Geophys., 20, 955-964, 2013

www.nonlin-processes-geophys.net/20/955/2013/

doi:10.5194/npg-20-955-2013

(c) Author(s) 2013. CC Attribution 3.0 License.

\title{
Using ensemble data assimilation to forecast hydrological flumes
}

\author{
I. Amour, Z. Mussa, A. Bibov, and T. Kauranne \\ Lappeenranta University of Technology, Lappeenranta, Finland \\ Correspondence to: I. Amour (idrissa.amour@lut.fi)
}

Received: 29 May 2013 - Revised: 23 September 2013 - Accepted: 9 October 2013 - Published: 8 November 2013

\begin{abstract}
Data assimilation, commonly used in weather forecasting, means combining a mathematical forecast of a target dynamical system with simultaneous measurements from that system in an optimal fashion. We demonstrate the benefits obtainable from data assimilation with a dam break flume simulation in which a shallow-water equation model is complemented with wave meter measurements. Data assimilation is conducted with a Variational Ensemble Kalman Filter (VEnKF) algorithm. The resulting dynamical analysis of the flume displays turbulent behavior, features prominent hydraulic jumps and avoids many numerical artifacts present in a pure simulation.
\end{abstract}

\section{Introduction}

Hydrological flumes and other phenomena related to rivers, estuaries, canals, and other water bodies that lend themselves to a one- or two-dimensional description have received somewhat less attention in computational fluid dynamics (CFD) research than flows in industrial processes, or fully threedimensional flows in general. However, there is one notable exception, namely, weather forecasting.

Weather models are complex codes that have received a lot of attention from the CFD community since the 1950s. Many techniques employed in weather forecasting are naturally amenable to other hydrostatic flows, such as river, estuary, and canal flows, but have yet to be tried in these applications. One of the most prominent of such techniques is data assimilation.

In the current paper, we introduce data assimilation of wave meter data into a river model that was originally presented by Martin and Gorelick (2005). Data assimilation is a process that optimally combines model forecasts with observations. In weather forecasting, data assimilation is used to generate the initial conditions for an ensuing forecast, but also to continuously correct a forecast towards observations, whenever these are available in the course of the forecast.

Turbulence, which is an irregular motion found in fluids (liquids and gases) when passing objects or streamlines of itself passing one another (Goldstein, 1938), is the main reason that makes the CFD model ambitious to assimilate, as we hope for more information from the available measurements to shape the behavior of the model estimates.

In the paper of Martin and Gorelick (2005), the authors present a shallow-water model of a dam break experiment conducted in a laboratory. The goal of the model is to simulate the behavior of the flume in the case that a dam suddenly breaks. Results from numerical simulations are compared with measurements by wave meters and pressure transducers that record water height during the experiment.

In the current article, we take the model and the measurements into account simultaneously in a process of continuous data assimilation. This results in a more realistic representation of the behavior of the flume during the experiment, in the sense that the resulting flume displays turbulent behavior, features prominent hydraulic jumps and avoids many numerical artifacts present in a pure simulation. As our data assimilation algorithm, we have chosen a recent version of the Ensemble Kalman Filter, the Variational Ensemble Kalman Filter (VEnKF) introduced by Solonen et al. (2012). Ensemble Kalman filters have the distinct advantage over other data assimilation methods that they can be implemented as "wrappers" on top of existing CFD codes without necessitating modification of the models themselves. Moreover, they conduct data assimilation continuously, so that their results can be compared with direct numerical simulations.

This paper is organized as follows. In the second section we discuss previous attempts at numerically simulating river flow. Section three describes the MODFreeSurf2D shallowwater code used in the data assimilation experiments introduced in Martin and Gorelick (2005). Section four discusses 
data assimilation and describes how it was implemented in the current research effort. Section five describes the dam break experiment and the way data assimilation was modified to accommodate the model and the data in this case. Section six describes the results of numerical tests, both with and without data assimilation. Section seven concludes the paper.

\section{Numerical simulation of river flows}

Simulation of river flows presents challenges to computational schemes due to the complex geometry and meandering path of the flow. Different schemes have been suggested and applied to try to capture as much information about the river as possible. A two-dimensional finite-element solution has been used to simulate an $11 \mathrm{~km}$ long reach of River Culm in Devon, UK, modeled by two-dimensional depth-averaged Reynolds equations (Bates and Anderson, 1993). The numerical model simulation finds an error of $\pm 2 \%$ in continuity, although the mass conservation is still adequate.

The same method has been applied to flow simulations in Aliparast (2009). The governing equations of the model are 2-D shallow-water equations, where the stress term is ignored because of the influence of bottom roughness caused by turbulent shear stress between grids (Yoon and Kang, 2004). The numerical model has been validated by an example of an oblique hydraulic jump for which an analytical solution is available (Aliparast, 2009). The numerical model has been tested with a dam break case in a converging-diverging flume (Bellos et al., 1991). The flume is $20.7 \mathrm{~m}$ long and $1.40 \mathrm{~m}$ wide, it has 5 stations used to record the water depth, and a dam is located $8.5 \mathrm{~m}$ from the closed end. The results of the dam break case simulation match well with the experimental results (Aliparast, 2009). However, in station 4, which is $13.5 \mathrm{~m}$ downstream from the dam, water depth is underestimated.

The finite volume method is a further widely-used numerical scheme. It has been applied for a shallow water model in Heniche et al. (2000), Zhang and Wu (2011) and Ying et al. (2009).

A recent application on the dam break case is presented by Baghlani (2011), where a robust flux vector splitting (FVS) scheme is applied. FVS has been frequently applied in solving similar compressible flow problems (e.g. in Baghlani, 2011; Erpicum et al., 2010; Toro and Vazquez-Cendon, 2012). Two well-known FVS methods are that of Steger and Warming and that of Van Leer (Drikakis and Tsangaris, 1993). Steger and Warming's FVS exploits the homogeneous property of the Euler equation and splits the fluxes into positive and negative parts with respect to the propagation (Drikakis and Tsangaris, 1993). Van Leer's FVS constructs the fluxes as a function of the local Mach number (Drikakis and Tsangaris, 1993). The FSV proposed by Baghlani (2011) decomposes the flux vector into positive and negative components by means of Jacobian matrices of the flux vectors and a Liou-Steffen splitting for decomposing the pressure term. The FVS has been criticized for its expensive computational cost, as the eigensystem of equations must be evaluated at every time step (Baghlani, 2011).

One approach to studying flow behavior is to construct flume properties directly from measurements by regression. A method of surface analysis and velocity changes of this type has been presented (Barcena et al., 2012). It uses regression with respect to a collection of model scenarios to form a continuous function of hydrodynamic responses. The method has been successful in predicting estuarine free surface and velocity with significant savings in computational cost for a short- and medium-term simulation period. One advantage of this method is its ability to simulate a long-term hydrodynamic flow with a short computational time.

Pure 2-D simulations of hydrological flows suffer from several handicaps. Because the numerical flow is twodimensional, it cannot capture the true three-dimensional flow, in particular turbulence: the numerical solution remains in perpetual hydrostatic balance. Even more importantly, the numerical time-stepping scheme implies that a flow front in front of a discontinuity, such as a flood wave, will only propagate one grid line per time step. The speed of this shock wave is therefore dependent on grid size and the numerical time step, and not on the correct physical speed. Finally, there is no way to connect the simulated flow to the true flow after the initial condition has been fixed. With data assimilation, we hope to address all three defects.

\section{MODFreeSurf2D}

MODFreeSurf2D is an open Matlab code that is designed to solve depth-averaged shallow-water equations (Martin and Gorelick, 2005). The code implements the semi-implicit, semi-Lagrangian time-stepping scheme of Casulli and Cheng (1992) and Casulli (1999), and uses a finite volume discretization. The scheme is stable and can simulate water/land boundaries (Martin and Gorelick, 2005; Casulli and Cheng, 1992).

\subsection{Depth-Averaged Shallow-Water Equations (DASWE)}

The governing equations in MODFreeSurf2D are the depthaveraged shallow-water equations as given in Martin and Gorelick (2005):

$$
\begin{aligned}
& \frac{\partial U}{\partial t}+U \frac{\partial U}{\partial x}+V \frac{\partial U}{\partial y}=-g \frac{\partial \eta}{\partial x}+\varepsilon\left(\frac{\partial^{2} U}{\partial x^{2}}+\frac{\partial^{2} U}{\partial y^{2}}\right) \\
& +\gamma_{T} \frac{\left(U_{a}-U\right)}{H}-g \frac{\sqrt{U^{2}+V^{2}}}{C_{z}^{2}} U+f V,
\end{aligned}
$$




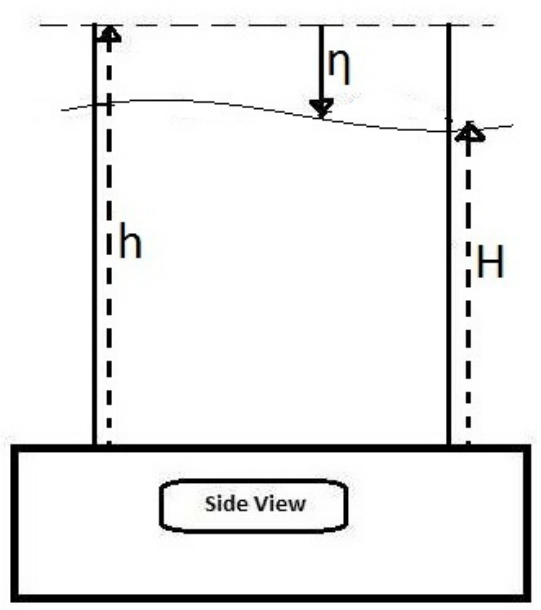

Fig. 1. ModFreeSurf2D variable definition (side view) which shows the relationship between free surface elevation $\eta$, total water depth $H$, and undisturbed water depth $h$ (Martin and Gorelick, 2005).

$$
\begin{aligned}
& \frac{\partial V}{\partial t}+U \frac{\partial V}{\partial x}+V \frac{\partial V}{\partial y}=-g \frac{\partial \eta}{\partial y}+\varepsilon\left(\frac{\partial^{2} V}{\partial x^{2}}+\frac{\partial^{2} V}{\partial y^{2}}\right) \\
& +\gamma_{T} \frac{\left(V_{a}-V\right)}{H}-g \frac{\sqrt{U^{2}+V^{2}}}{C_{z}^{2}} V-f U, \\
& \frac{\partial \eta}{\partial t}+\frac{\partial(H U)}{\partial x}+\frac{\partial(H V)}{\partial y}=0,
\end{aligned}
$$

where $U$ is the depth-averaged $x$ direction velocity component, $V$ is the depth-averaged $y$ direction velocity component, $\eta$ is the free surface elevation, $g$ is the gravitational constant, $t$ is time, $\varepsilon$ is the horizontal eddy viscosity, $f$ is the Coriolis parameter, $H=h+\eta$ is the total water depth, $\gamma_{T}$ is the wind stress coefficient, $C_{z}$ is the Chezy coefficient, and $U_{a}$ and $V_{a}$ are wind velocities. In the above, $h$ is the undisturbed water depth. Figure 1 illustrates the variable definitions of MODFreeSurf2D.

Top friction and bottom friction boundaries are given by Eqs. (4) and (5), respectively.

$$
\begin{aligned}
& \nu \frac{\partial U}{\partial z}=\gamma_{T}\left(U_{a}-U\right), \quad \nu \frac{\partial V}{\partial z}=\gamma_{T}\left(V_{a}-V\right) \\
& \nu \frac{\partial U}{\partial z}=g \frac{\sqrt{U^{2}+V^{2}}}{C_{z}^{2}} U, \quad \nu \frac{\partial V}{\partial z}=g \frac{\sqrt{U^{2}+V^{2}}}{C_{z}^{2}} V,
\end{aligned}
$$

where $v$ is the kinetic viscosity coefficient, and $z$ indicates the vertical direction (Martin and Gorelick, 2005).

\subsection{Numerical approximation}

In MODFree2DSurf a combination of a semi-implicit, semiLagrangian time-stepping scheme and a finite-volume discretization is employed to numerically solve the hydrological shallow-water equations on a rectangular grid. This scheme provides a stable solution, even for a time step larger than the Courant-Friedrichs-Lewy (CFL) restriction defined by

$\mathrm{CFL}=w \frac{\Delta t}{\Delta x_{i}}$,

where $w$ is the velocity component in the $x_{i}$ direction, $i=$ $1,2, \Delta t$ is the time step size, and $\Delta x_{i}$ is the cell dimension in the $x_{i}$ direction of flow (Martin and Gorelick, 2005). The CFL relates fluid velocity and time step size to computational cell size, and requires that it should be smaller than 1 (Martin and Gorelick, 2005).

\subsubsection{Semi-implicit representation}

In this representation, the free surface elevation $\eta$ and the horizontal velocity components $U$ and $V$ are the unknown variables at time $N+1$ :

$$
\begin{aligned}
\eta_{i, j}^{N+1}= & \eta_{i, j}^{N}-\theta \frac{\Delta t}{\Delta x}\left(H_{i+1 / 2, j}^{N} U_{i+1 / 2, j}^{N+1}-H_{i-1 / 2, j}^{N} U_{i-1 / 2, j}^{N+1}\right) \\
& -\theta \frac{\Delta t}{\Delta x}\left(H_{i, j+1 / 2}^{N} V_{i, j+1 / 2}^{N+1}-H_{i, j-1 / 2}^{N} V_{i, j-1 / 2}^{N+1}\right) \\
& -(1-\theta) \frac{\Delta t}{\Delta x}\left(H_{i+1 / 2, j}^{N} U_{i+1 / 2, j}^{N}-H_{i-1 / 2, j}^{N} U_{i-1 / 2, j}^{N}\right) \\
& -(1-\theta) \frac{\Delta t}{\Delta x}\left(H_{i, j+1 / 2}^{N} V_{i, j+1 / 2}^{N}-H_{i, j-1 / 2}^{N} V_{i, j-1 / 2}^{N}\right),
\end{aligned}
$$

$$
\begin{aligned}
U_{i+1 / 2, j}^{N+1}= & F U_{i+1 / 2, j}^{N}-(1-\theta) \frac{g \Delta t}{\Delta x}\left(\eta_{i+1, j}^{N}-\eta_{i, j}^{N}\right) \\
& -\theta \frac{g \Delta t}{\Delta x}\left(\eta_{i+1, j}^{N+1}-\eta_{i, j}^{N+1}\right)+\Delta t \frac{\gamma_{T}\left(U_{a}-U_{i+1 / 2, j}^{N+1}\right)}{H_{i+1 / 2, j}^{N}} \\
& -g \Delta t \frac{\sqrt{\left(U_{i+1 / 2, j}^{N}\right)^{2}+\left(V_{i+1 / 2, j}^{N}\right)^{2}}}{C z_{i+1 / 2, j}^{2} H_{i+1 / 2, j}^{N}} U_{i+1 / 2, j}^{N+1}, \quad(8) \\
V_{i, j+1 / 2}^{N+1}= & F V_{i, j+1 / 2}^{N}-(1-\theta) \frac{g \Delta t}{\Delta y}\left(\eta_{i, j+1}^{N}-\eta_{i, j}^{N}\right) \\
& -\theta \frac{g \Delta t}{\Delta y}\left(\eta_{i, j+1}^{N+1}-\eta_{i, j}^{N+1}\right)+\Delta t \frac{\gamma_{T}\left(V_{a}-V_{i, j+1 / 2}^{N+1}\right)}{H_{i, j+1 / 2}^{N}} \\
& -g \Delta t \frac{\sqrt{\left(U_{i, j+1 / 2}^{N}\right)^{2}+\left(V_{i, j+1 / 2}^{N}\right)^{2}}}{C z_{i, j+1 / 2}^{2} H_{i, j+1 / 2}^{N}} V_{i, j+1 / 2}^{N+1} . \quad \text { (9) }
\end{aligned}
$$

In the above equations, $\Delta x$ is the computational volume length in the $x$ direction, $\Delta y$ is the computational volume length in the $y$ direction, and $\Delta t$ is the computational time step (Martin and Gorelick, 2005). The parameter $\theta$ dictates the degree of implicitness of the solution. Its value ranges between 0.5 and 1 , where $\theta=0.5$ means that the approximation is centered in time and $\theta=1.0$ means that the approximation is completely implicit (Casulli and Cheng, 1992). 
The operators $F U$ and $F V$ in Eqs. (8) and (9) contain the advective, viscous, and Coriolis components of the governing equations (Martin and Gorelick, 2005).

The value of the Chezy coefficient in Eq. (10) is given in terms of Manning's roughness coefficient $M n$, which is assumed to be dimensionless (Martin and Gorelick, 2005). Other details of the discretization process can be found in Martin and Gorelick (2005):

$C_{z_{i+1 / 2, j}}=\frac{\left(H_{i+1 / 2, j}\right)^{1 / 6}}{M n_{i+1 / 2, j}}$.

\subsection{The boundary condition}

The model itself identifies the location of water/land boundaries using the following equations (Martin and Gorelick, 2005):

$H_{i+1 / 2, j}^{N+1}=\max \left(0, h_{i+1 / 2, j}+\eta_{i, j}^{N+1}, h_{i+1 / 2, j}+\eta_{i+1, j}^{N+1}\right)$,

$H_{i, j+1 / 2}^{N+1}=\max \left(0, h_{i, j+1 / 2}+\eta_{i, j}^{N+1}, h_{i, j+1 / 2}+\eta_{i, j+1}^{N+1}\right)$.

Two types of horizontal boundary conditions have been set:

(i) The projection of the velocity normal to the domain boundary:

$\frac{\partial U}{\partial t}+U_{\text {upw }} \frac{\partial U}{\partial n}=0$,

where $U_{\text {upw }}$ is the upwinded normal direction velocity component, and $n$ is the direction normal to the domain boundary (Martin and Gorelick, 2005).

(ii) To limit wave reflections at open boundaries, the following condition is imposed:

$\frac{\partial \eta}{\partial t}+C_{n} \frac{\partial \eta}{\partial n}=0$,

where $C_{n}$ is the propagation velocity from grid points around the boundary (Martin and Gorelick, 2005).

\section{Data assimilation}

\subsection{Overview}

Data assimilation aims to establish an optimal compromise between the prediction of a computational model and a set of observations. Both the model and the observations are assumed to be incorrect and contain some error. Heuristically, data assimilation takes some weighted average between these two estimates, with weights inversely proportional to the anticipated error in each (Daley, 1991).

Two forms of data assimilation have been common in weather forecasting. In "lumped" data assimilation, the goal is to produce a single initial state for the system to be simulated, from which a subsequent forecast can be launched. In "lumped" data assimilation it has been possible to impose the model state equation - a set of conservation laws - exactly on that initial state, especially when so-called variational assimilation has been used (Le Dimet and Talagrand, 1986; Lewis and Derber, 1985; Courtier and Talagrand, 1987). However, variational data assimilation implicitly contains the same defects that the model it is based on contains - for example some model bias. Bias threatens to throw the model trajectory away from the true physical flow, which is tracked by observations even when they contain some noise.

In continuous data assimilation, on the other hand, the solution of the state equation is constantly "nudged" towards observations (Lorenc, 1986). This means that the state equation is only approximately true and that several conservation properties may get lost, but the model trajectory is likely to always stay close to the true observed trajectory.

The Extended Kalman Filter (EKF) (Kalman, 1960) combines the best properties of both lumped and continuous data assimilation methods. Unfortunately, the computational complexity of the classical EKF is prohibitively high for high-dimensional numerical models such as those appearing in geophysical simulations. However, this situation has started to change with the emergence of Ensemble Kalman filters (EnKF), (Evensen, 1994). Ensemble Kalman filters are stochastic approximations of the Extended Kalman Filter that purport to preserve many of its good properties. In practice, the degree to which this is achieved depends crucially on the particular EnKF variant chosen.

Some variants of Ensemble Kalman filters draw their inspiration from the same Bayesian paradigm as the original Kalman Filter does. A prominent example of these methods is the maximum likelihood estimation filter (MLEF) introduced in Zupanski (2004). MLEF solves a Bayesian minimization with an ensemble of forecasts and uses the Limited Memory BFGS method to minimize a cost function that measures the distance of observations from the forecast. However, it generates a single ensemble of forecasts in the beginning of the forecast and uses it for all the minimzations, unlike the Variational Ensemble Kalman Filter (VEnKF) that will be introduced below. As will become evident from the convergence behavior of VEnKF, re-sampling the ensemble very frequently dramatically improves the convergence of the method and the stability of the corresponding Kalman filter.

\subsection{VEnKF}

The Variational Ensemble Kalman Filter (VEnKF) is a stochastic approximation of the EKF. In this paper, we restrict ourselves to a brief discussion of the main ideas behind the VEnKF. A more detailed discussion can be found in Solonen et al. (2012). We begin by introducing the following coupled system of stochastic dynamic equations:

$$
\begin{aligned}
s_{k+1} & =\mathcal{M}_{k}\left(s_{k}\right)+\varepsilon_{k}, \\
o_{k+1} & =\mathcal{H}_{k+1}\left(s_{k+1}\right)+\zeta_{k+1},
\end{aligned}
$$


where $\mathcal{M}_{k}$ is an $N$-dimensional transition operator used to forecast the model state at time instance $k+1$ given the model state at time instance $k ; \mathcal{H}_{k+1}$ is an observation operator that maps the model state $s_{k+1}$ to the observation $o_{k+1} ; \varepsilon_{k}$ and $\zeta_{k+1}$ are zero mean random terms that define prediction and observation error, respectively. Our task is to define an estimate for $s_{k+1}$ given the operators $\mathcal{M}_{k}, \mathcal{H}_{k+1}$, the observation $o_{k+1}$, and the covariance matrices of $\varepsilon_{k}$ and $\zeta_{k+1}$, hereafter defined as $C_{\varepsilon_{k}}$ and $C_{\zeta_{k+1}}$. In many cases, $\varepsilon_{k}$ and $\zeta_{k+1}$ are also assumed to be normally distributed, although this requirement can be relaxed.

The motivation behind the VEnKF is quite similar to that of the EnKF. Foremost, we compute a sample estimate for the prior covariance, where the samples are explicitly generated by Eq. (15). Thereafter, instead of following the EKF formulas as is done in EnKF, we replace them with a MAP (maximum a posteriori probability) estimate problem. By taking $-\log$ of the MAP cost function we arrive at an equivalent minimization problem as suggested in Auvinen et al. (2009):

$$
\begin{aligned}
l\left(s \mid o_{k+1}\right)= & \frac{1}{2}\left(s-s_{k+1}^{\mathrm{p}}\right)^{T}\left[C_{k+1}^{\mathrm{p}}\right]^{-1}\left(s-s_{k+1}^{\mathrm{p}}\right)+ \\
& \left.\frac{1}{2}\left(o_{k+1}-\mathcal{H}_{k+1}(s)\right)^{T} C_{\zeta_{k+1}}^{-1}\left(o_{k+1}-\mathcal{H}_{k+1}(s)\right)\right) .
\end{aligned}
$$

Here $s_{k+1}^{\mathrm{p}}$ is the predicted model state at time instance $k+1$ and $C_{k+1}^{\mathrm{p}}$ is the covariance matrix of the prediction. When transition and observation operators are linear, it can be proven (Simon, 2006) that a minimum variance unbiased estimator for $s_{k+1}$, hereafter denoted as $s_{k+1}^{\text {est }}$, minimizes Eq. (17), whereas the covariance matrix of this estimate, which we denote by $C_{k+1}^{\text {est }}$, is defined by the inverse Hessian of Eq. (17). This approach can also be expanded to non-linear cases.

Before giving a rigorous formulation of the VEnKF algorithm, we need to introduce some supporting notation. Let $\left\{e_{k, i}\right\}_{i=1}^{N}$ denote an ensemble of cardinality $N$ sampled from the distribution of $s_{k}^{\text {est }}$. More precisely, $\forall i e_{k, i} \sim$ $\mathcal{N}\left(s_{k}^{\text {est }}, C_{k}^{\text {est }}\right)$. In addition, we denote the mean of $\left\{e_{k, i}\right\}_{i=1}^{N}$ by $\bar{e}_{k}$ and introduce an $M$-element vector $X\left(e_{k, i}\right)$ defined as follows:

$X\left(e_{k, i}\right)=\left(\left(e_{k, 1}-\bar{e}_{k}\right), \ldots,\left(e_{k, N}-\bar{e}_{k}\right)\right) / \sqrt{N-1}$.

The VEnKF algorithm now reads as follows:

i. Compute the model prediction: $s_{k+1}^{\mathrm{p}}=\mathcal{M}_{k}\left(s_{k}\right)$.

ii. Move the ensemble $\left\{e_{k, i}\right\}_{i=1}^{N}$ forward using Eq. (15):

$$
\forall i \tilde{e}_{k+1, i}=\mathcal{M}_{k}\left(e_{k, i}\right)
$$

iii. Define the sample estimate for the prior covariance:

$$
C_{k+1}^{\mathrm{p}}=X\left(\tilde{e}_{k+1, i}\right)\left(X\left(\tilde{e}_{k+1, i}\right)\right)^{T}+C_{\varepsilon_{k}} .
$$

iv. Assign $s_{k+1}^{\text {est }}$ to the minimizer of the cost function (17) and $C_{k+1}^{\text {est }}$ to an approximation of the inverse Hessian of Eq. (17).

v. Update the ensemble $\left\{\tilde{e}_{k+1, i}\right\}_{i=1}^{N}$ by sampling from $\mathcal{N}\left(s_{k+1}^{\text {est }}, C_{k+1}^{\text {est }}\right)$.

The strength of the VEnKF is that it allows a memoryefficient representation of the prior covariance $C_{k+1}^{\mathrm{p}}$. The latter is advantageous when the model state dimension is too big to allow the explicit storage of the covariance matrices. However, in order to implement steps (iv) and (v) of the presented algorithm, we need to evaluate the cost function defined by Eq. (17) and specify a low-memory approximation for its inverse Hessian. The first goal is achieved by leveraging the Sherman-Morrison-Woodbury formula to invert $C_{k+1}^{\mathrm{p}}$. More precisely:

$$
\begin{aligned}
& {\left[C_{k+1}^{\mathrm{p}}\right]^{-1}=\left[C_{\varepsilon_{k}}+X\left(\tilde{e}_{k+1, i}\right)\left(X\left(\tilde{e}_{k+1, i}\right)\right)^{T}\right]^{-1}=C_{\varepsilon_{k}}^{-1}} \\
& -C_{\varepsilon_{k}}^{-1} X\left(\tilde{e}_{k+1, i}\right)\left(I+\left(X\left(\tilde{e}_{k+1, i}\right)\right)^{T} C_{\varepsilon_{k}}^{-1} X\left(\tilde{e}_{k+1, i}\right)\right)^{-1} \times \\
& \left(X\left(\tilde{e}_{k+1, i}\right)\right)^{T} C_{\varepsilon_{k}}^{-1} .
\end{aligned}
$$

Formulation (18) can be directly inserted into cost function (17), so it is not necessary to store the full matrices in order to evaluate it. Since the matrix $C_{\varepsilon_{k}}$ is usually specified by a simplified (diagonal or block-diagonal) structure, and the ensemble size is assumed to be much smaller than the model state dimension, the inversion operations in Eq. (18) are feasible. Reverting back to the implementation of step (v) of the VEnKF algorithm, we suggest approximating the inverse Hessian of Eq. (17) by either the full rank low-memory representation employed by the L-BFGS unconstraint optimizer (Nocedal and Wright, 1999) or the reduced rank representation in Krylov space generated by conjugate gradient minimization of Eq. (17) (Bardsley et al., 2013).

The computational complexity of the VEnKF algorithm remains linear in the number of degrees of freedom of the model despite the fact that it solves a minimization problem with observations every time step. This follows from the fact that the minimization problem is very well-conditioned and converges in a small number of iterations. This number is likely to remain independent of the number of degrees of freedom, because the minimization in VEnKF is identical to that applied in three-dimensional variational data assimilation that has been observed to have this behavior in operational weather data assimilation. This good behavior comes from the fact that in the current application scenario the assimilation window is just one time step long, and therefore the Hessian matrix remains diagonally dominated and the initial guess very good, in the same manner as in the minimization applied in implicit time-stepping schemes. The convergence history of the residual of minimization indeed shows fast linear convergence, as seen in Fig. 2. 


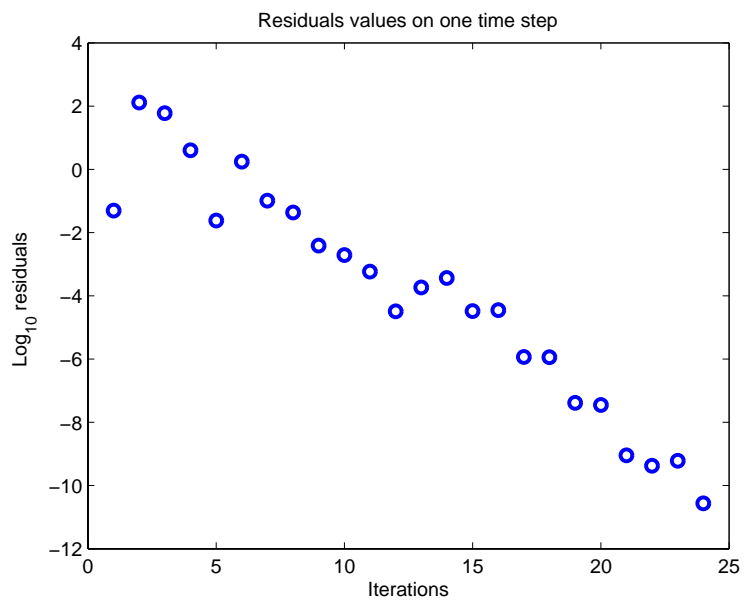

Fig. 2. Sample residuals plotted for one time step.

\section{Dam break experiments}

One of the applications published in Martin and Gorelick (2005) is a dam break experiment. The experiment consists of a flume $21.2 \mathrm{~m}$ long and $1.4 \mathrm{~m}$ wide. The flume is closed at one end and open at the other end. It has a curved constriction $5.0 \mathrm{~m}$ from the closed end that ends $4.7 \mathrm{~m}$ from the open end. A dam is located $8.5 \mathrm{~m}$ from the closed end, with an opening of width $0.6 \mathrm{~m}$. The flume has a slope of 0.002 with water at a height of $0.15 \mathrm{~m}$ behind the dam (Martin and Gorelick, 2005).

Wave meters and pressure transducers were located at 8 locations, as seen in Fig. 3; however, the water depth measurements were only given in seven locations. The recorded water depths last for $62 \mathrm{~s}$ after the dam is broken. With the dam position chosen as the origin, the wave meters are located at places $x=-8.5,-4.5$, and $-0.0 \mathrm{~m}$, and the pressure transducers are placed at $x=+0.0,+2.5,+5.0,+7.5$, and $+10.0 \mathrm{~m}$ (Martin and Gorelick, 2005). The computational time step used in the experiment is $\Delta t=0.103 \mathrm{~s}$ and the grid dimensions are $\Delta x=0.05 \mathrm{~m}$ and $\Delta y=0.125 \mathrm{~m}$. With this grid cell size, the geometry is sliced into $30 \times 171$ grid cells. Finally, simulated water heights at the seven locations were compared with heights measured by the wave meters and pressure transducers.

\subsection{VEnKF parameters}

The state vector for the assimilation is defined as the vector of heights at the center of a grid point. The complete state vector comprises the free surface elevation $\eta$ and the horizontal velocities $u$ in the $x$ direction and $v$ in the $y$ direction for the entire domain, i.e., $s=\left[\begin{array}{lll}l & u & v\end{array}\right]^{T}$. The model has, therefore, altogether 16000 spatial degrees of freedom. With the interpolations described in Sect. 5.3, the ensembles are sampled in every time step of the assimilation. The observation error and the model error covariance matrices are both assumed

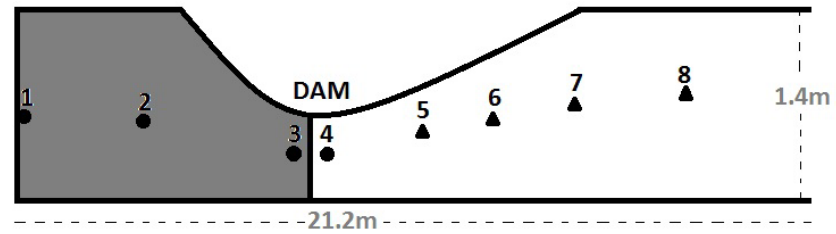

Fig. 3. Plan view flume layout for the dam break experiment of Bellos et al. (1992). Wave meter locations are displayed with circles. Pressure transducer locations are displayed with triangles (Martin and Gorelick, 2005).

to be diagonal. The observation operator $\mathcal{H}$ in Eq. (17) is a linear operator that maps the state vector to the observation space corresponding to all grid points covered by the interpolated data, but restricted to the water height values only.

\subsection{Experiment 1: VEnKF application to dam break with synthetic data}

The aim of this experiment is to examine both qualitative and quantitative characteristics of the VEnKF method to the dam break experiment. The data set has been generated by adding normally distributed noise with mean 0 and a variance of $5 \times$ $10^{-2}$ from the solution of the model simulation. To make the experiment more realistic, data has been picked in 8 positions corresponding to wave meter locations defined in Martin and Gorelick (2005). More precisely, the time interval between the data in all locations was fixed, but chosen randomly for every location. This setup emulates the fact that wave meters do not necessarily collect information at the same time.

\subsubsection{Results for experiment 1}

In Figs. 4 and 5, the matching between the data, VEnKF estimates (50 ensembles) and the model simulation, here referred to as the truth, is shown for all 8 wave meter locations. The target of the current study is not really data assimilation for the purpose of a subsequent forecast with VEnKF, as would be the case in an atmospheric dynamics context, but instead qualitatively better hind-casting of a catastrophic event such as a dam breaking down, with an ensemble-based approach. In our case, the length of each forecast is therefore just one computational time step at a time with interpolated observations. This close match between the model and observations over one time step also results in very compact ensemble spreads, as can be seen in Fig. 6 .

Figure 7 shows a plot of root mean square error (RMSE) for the entire duration of the simulation. The error first increases, then decreases steadily. Such error behavior can be explained by the fact that at the begining of the dam break, a steady initial state quickly breaks into a turbulent flume that then peters out to a drib as the water eventually runs out. In the forecast skill plot shown in Fig. 8, we have plotted the forecast skill from a time about $4 \mathrm{~s}$ after the dam break for 
(a): SensorNo.1

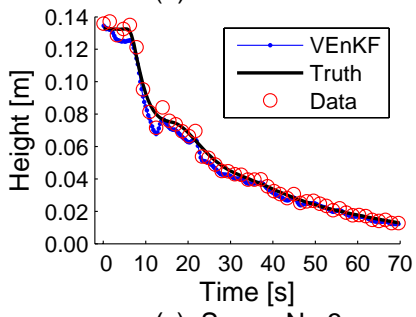

(c): SensorNo.3

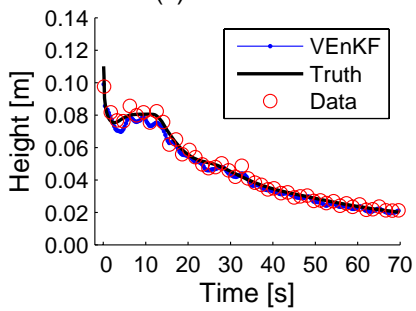

Fig. 4. Experiment 1: Comparison of VEnKF estimates, true water depth and data of the dam break experiment for the first four sensors at the upstream end.

a period of $10 \mathrm{~s}$ from the begining of the experiment, as this period is the best one to display the error before water has started to run out from the flume.

\subsection{Experiment 2: VEnKF for dam break experiment with real data}

The published data set (see Martin and Gorelick, 2005) of measurements is used to assimilate water heights. The high sparsity of the set of measurements is challenging for data assimilation. Observations come at an average rate of 1.6 observations in one or more locations per time step and at a maximum of 5 locations per time step. They feature only measurements of water height. This means that the number of observations in relation to the dimension of state space is approximately $1 / 100000$, since the computational time step is $0.1 \mathrm{~s}$. For this reason, the observations are interpolated in time by a spline scheme and in space by a Gaussian mask to make them dense enough for the VEnKF assimilation scheme. This means we interpolate the observations in time and extrapolate each of them in space by a Gaussian kernel. After this, the ratio of the number of observations to system dimension improves to $1 / 50$.

\subsubsection{Shore boundary definition for the VEnKF}

As can be seen from the MAP estimate problem (17), the VEnKF does not account for additional prior knowledge beyond the observations. This means that in the case of problems on bounded domains, there is no way to include information about the boundaries in the Kalman filter analysis. If the prediction model automatically maintains the boundaries in accordance with defined constraints, one can simply (a): SensorNo.5
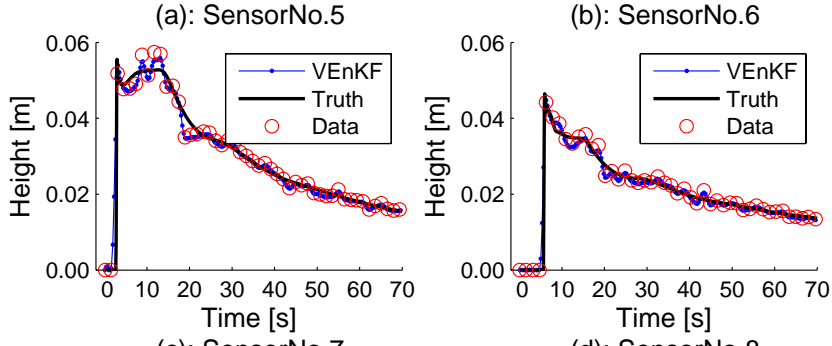

(c): SensorNo.7
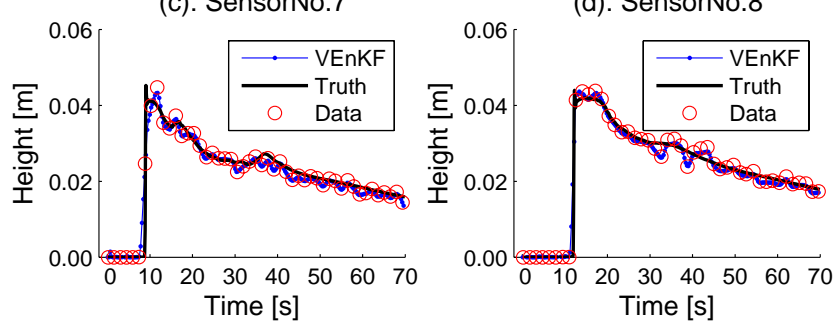

Fig. 5. Experiment 1: Comparison of VEnKF estimates, true water depth and data of the dam break experiment for the last four sensors.

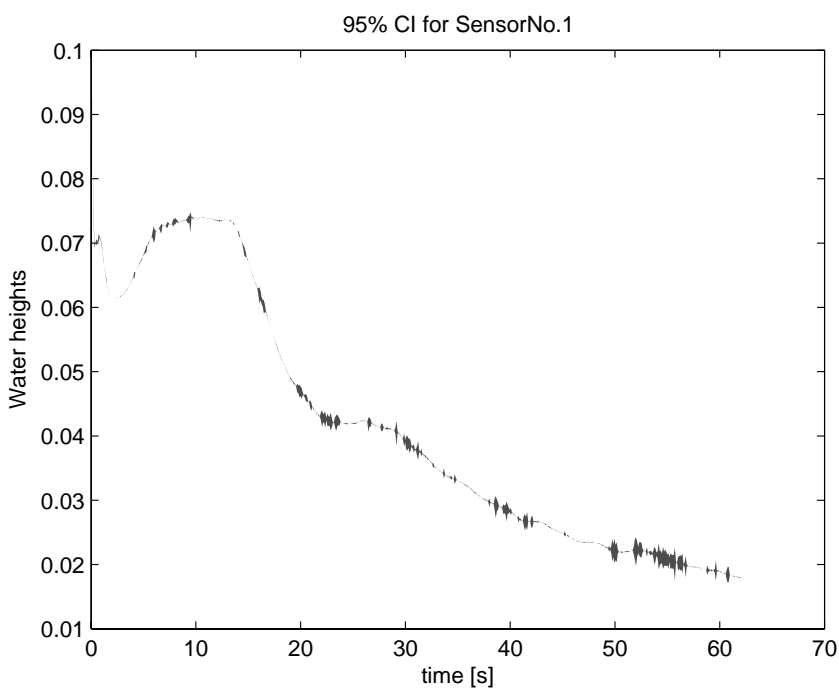

Fig. 6. $95 \%$ confidence region is shown for the location of Sensor No. 1.

reduce the data assimilation analysis to the inner part of the model domain. However, this approach is complicated when the boundaries change over time.

In our experiments, we use a strategy that allows us to account more flexibly for evolving boundaries, albeit without guaranteeing that the boundaries will be preserved exactly as required by the model constraints. Information about the boundaries is included in the model uncertainty description, i.e., in the model error covariance $C_{\varepsilon_{k}}$ (see Eq. 15). This changes the analytical representation of the boundaries to a probabilistic description. Thus, there is no absolute certainty about where the boundaries are located, but there is more confidence about the evolution of boundaries than that of the model. 


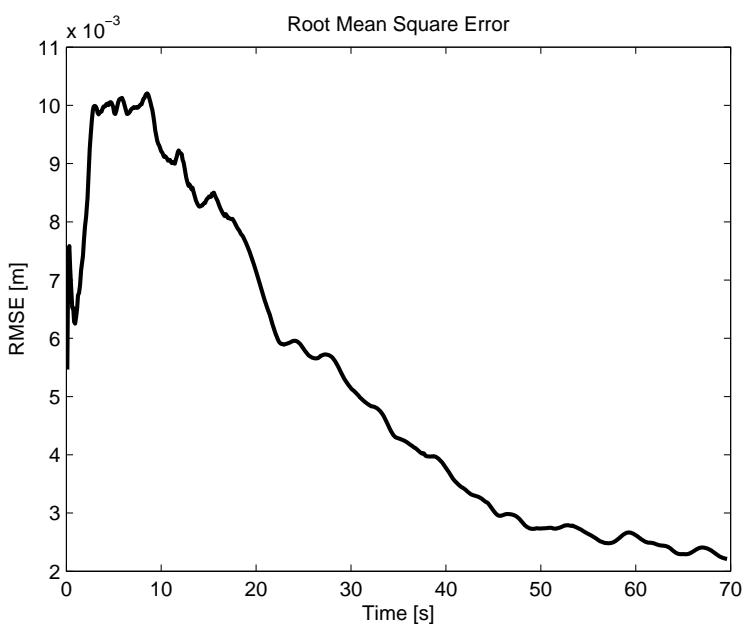

Fig. 7. The RMSE plot for the entire time of assimilation.

In the dam break case studied here, we have prior knowledge about the shoreline and there will be no water in places where there is a riverbank. Therefore, we define the model covariance $C_{\varepsilon_{k}}$ such that the state elements that are confined to the riverbank have variances much smaller than the variances assigned to the rest of the state. This strategy shifts the responsibility of maintaining the boundaries to the data assimilation analysis.

\section{Model applications with real data}

\subsection{Results without data assimilation}

The results of direct model simulations with MODFreeSurf2D show that the simulated water depth matches well with the measured depth only for the three measurement locations above the dam (at the upstream end), as can be seen in Fig. 9a-c. The results are less accurate for other locations below the dam due to the emergence of super-critical flows in the downstream end. The downstream end is also characterized by turbulent flow, and the model only tracks the height of water, but not the turbulent fine structure of the flow. In Fig. 9d as well as Fig. 10a and b in particular, we can see the discontinuity of the flow at the beginning of the dam opening.

\subsection{Results with data assimilation}

The model error and the observation error covariance matrices were set to $\mathbf{C}_{\varepsilon_{k}^{\mathrm{p}}}=(0.0011)^{2} \mathbf{I}$ and $\mathbf{C}_{\zeta k}=(0.001)^{2} \mathbf{I}$, respectively. We use the initial estimate of the state $x_{0}^{\text {est }}$ equals the initial height of water and the initial covariance estimate $C_{0}^{\text {est }}=\mathbf{I}$.

Measurements are incorporated into the model, and the assimilation is done with an ensemble size of 75 members. The number of LBFGS iterations and stored vectors is set to 25 . When the dam is removed, a strong flood wave is generated

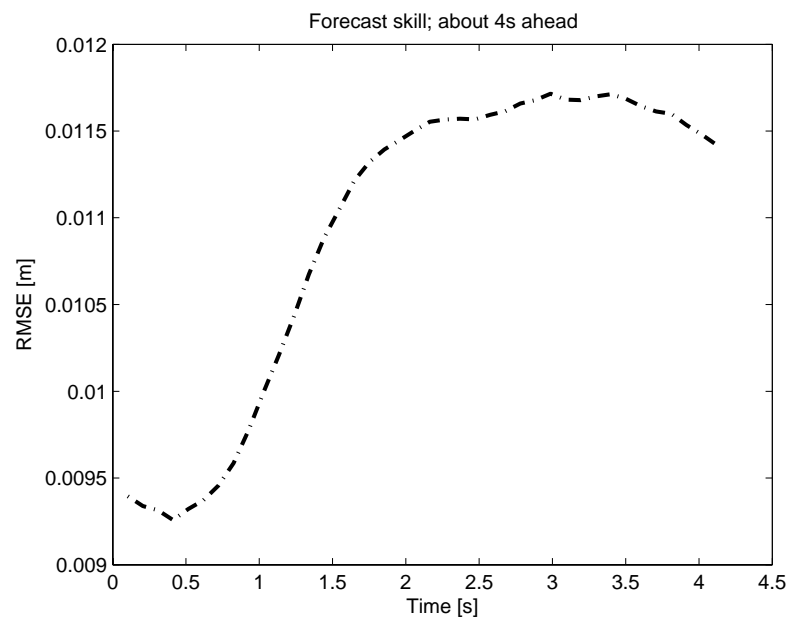

Fig. 8. The forecast skill plot for about $4 \mathrm{~s}$ period of forecast
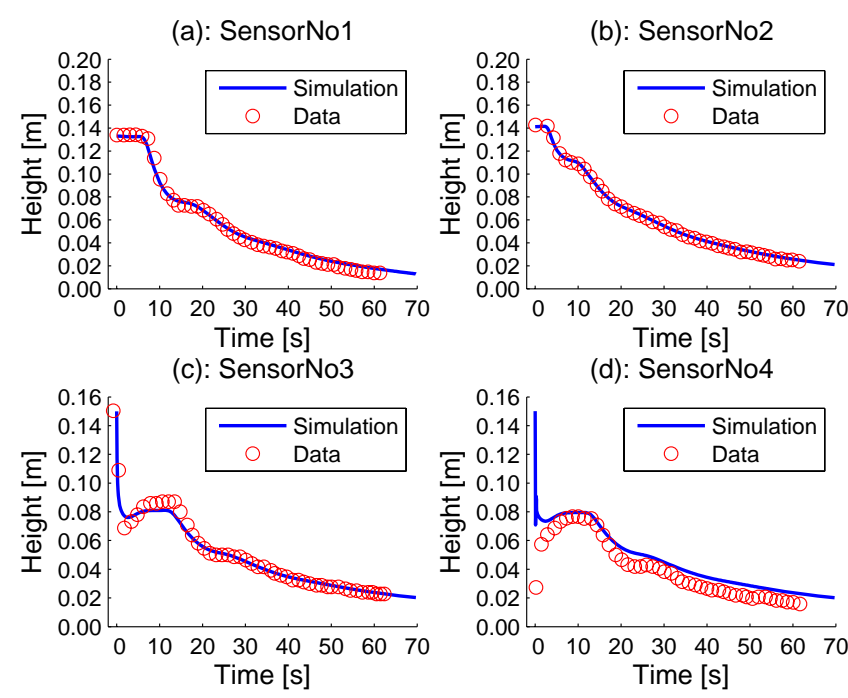

Fig. 9. Comparison of simulated water depth and measured depth of the dam break experiment for the first four sensors at the upstream end.

and propagated downstream from the flume. The variation of water depth with time is compared with experimental data as given by the seven sensors; see Figs. 11 and 12 .

From the results it can be seen that at the location immediately after the dam (location 4), the original simulation did not capture the behavior of the flow at the beginning of the simulation. However, VEnKF is able to approximate the water height and the structure of the flow. The same situation was observed in locations 5, 6 and 8, where the VEnKF result captures well the most prominent features of the flow.

At all seven sensors located at the upstream and downstream ends with available measurements, these measurements agree well with the VEnKF results. This demonstrates the capability and accuracy of the VEnKF for predicting dam break flows for rivers and streams. 
(a): SensorNo5

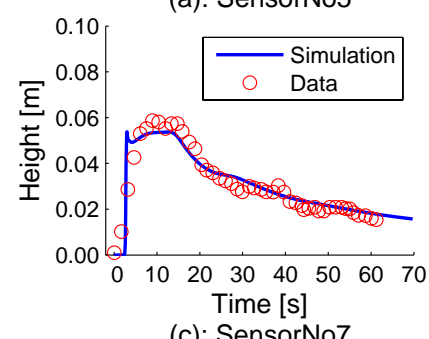

(c): SensorNo7
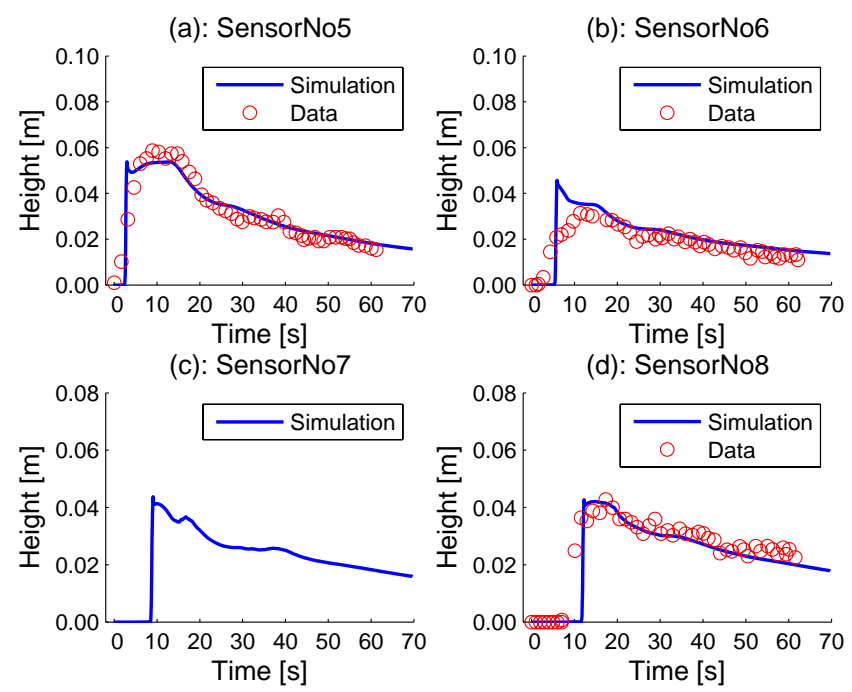

(d): SensorNo8

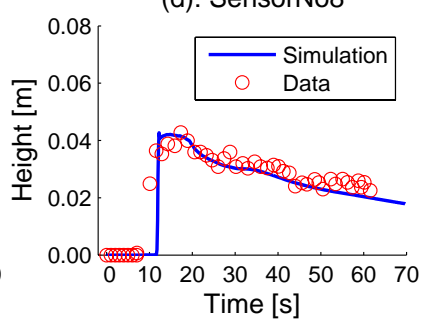

Fig. 10. Comparison of simulated water depth and measured depth of the dam break experiment for the last four sensors at the downstream end. Sensor No. 7 did not have measurements.

(a): SensorNo1

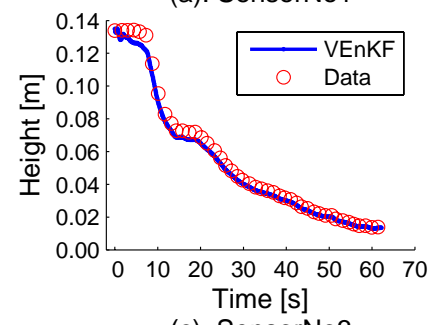

(c): SensorNo3

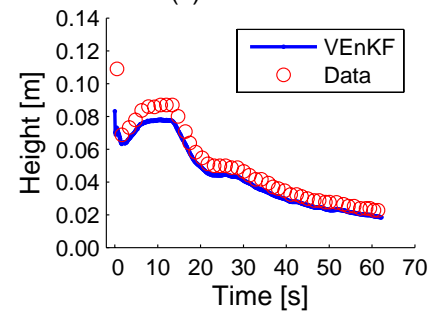

Fig. 11. Experiment 2: Comparison of VEnKF results and measured depth of the dam break experiment for the first four sensors at the upstream end.

It is worth pointing out the time series of water heights at sensor 7 that did not provide any measurements because of a sensor malfunction. If we compare the simulated curve in Fig. 10c with direct simulation to that of Fig. 12c with data assimilation, we see that the latter contains similar fine scale oscillations due to small waves as the sensors with observations, but that these oscillations are missing in Fig. 10c.

This demonstrates that the qualitative improvements towards a more realistic representation of the flume are not restricted to sites with observations, but are indeed spread throughout the computational domain. This can be seen in more detail in the accompanying videos that represent the
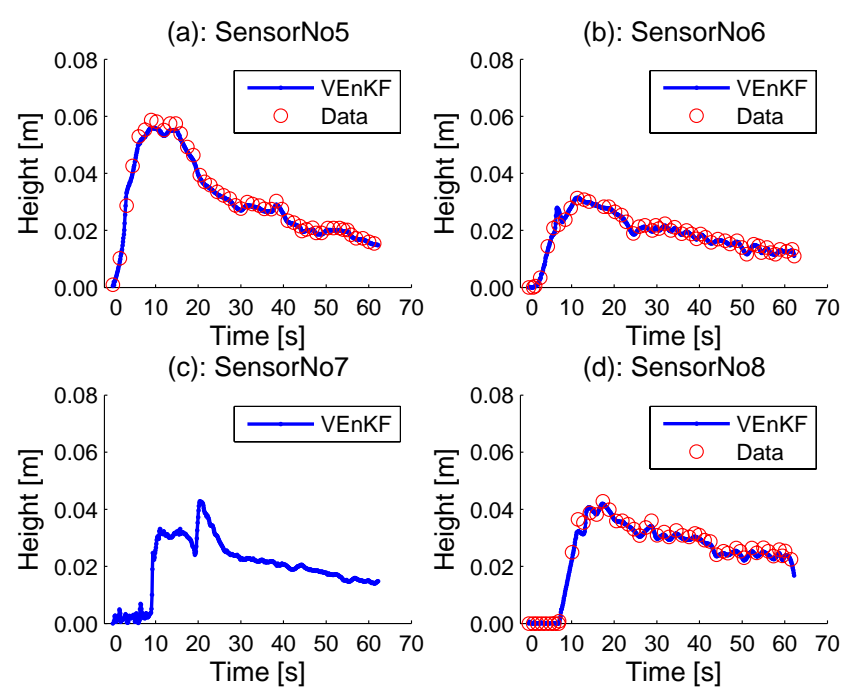

Fig. 12. Experiment 2: Comparison of VEnKF results and measured depth of the dam break experiment for the last four sensors at the downstream end. Sensor No. 7 did not have measurements.

pure simulation and the flume obtained with data assimilation.

\section{$7 \quad$ Discussion and conclusions}

The use of data assimilation to complement forecasts made by mathematical models with observational data is a growing trend in scientific computing. This trend is likely to continue, since the computer capacity accessible to researchers is increasing rapidly, and many different kinds of automatic measurement devices are becoming available that provide large numbers of measurements from target systems.

In the foregoing sections, we have demonstrated some of the benefits that data assimilation can bring to hydrological modeling. The resulting analysis of a dam break flume behaves in a more realistic manner than the corresponding computer simulation alone. It displays turbulent behavior such as the real flow, features prominent hydraulic jumps, and avoids several numerical artifacts.

Another benefit of data assimilation is a proper statistical treatment of flume simulations. Traditional mathematical models are deterministic, whereas in computer simulation we can only approximate a real physical phenomenon in a statistical sense. For this reason, the adoption of a version of Kalman filtering, the Variational Ensemble Kalman Filter (VEnKF), adds value to the simulation, as it automatically incorporates information about the expected error covariance of the analysis of the flume into the approximate error covariance matrix that it computes in the course of data assimilation. Continuous data assimilation therefore addresses qualitative defects in flow simulations and correctly interprets simulated numerical values as samples from a distribution of possible physical values, not as true physical values. 
Acknowledgements. Thanks to Lappeenranta University of Technology and the World Bank Project funding through the University of Dar es salaam and Dar es salaam University College of Education, without these funding this work would be impossible. Our thanks also go to the anonymous reviewers for their insightful review of our work.

Edited by: R. Buizza

Reviewed by: two anonymous referees

\section{References}

Aliparast, M.: Two-dimensional finite volume method for dambreak flow simulation, Int. J. Sediment Res., 24, 99-107, 2009.

Auvinen, H., Bardsley, J., Haario, H., and Kauranne, T.: The variational Kalman filter and an efficient implementation using limited memory BFGS, Int. J. Numer. Meth. Fl., 64, 314-335, 2009.

Baghlani, A.: Simulation of dam-break problem by a robust fluxvector splitting approach in Cartesian grid, Scientia Iranica, 18, 1061-1068, 2011.

Barcena, J. F., Garcia, A., Garcia, J., Alvares, C., and Revilla, J. A.: Surface analysis of free surface and velocity to changes in river flow and tidal amplitude on a shallow mesotidal estuary: An application in Suances Estuary (Nothern Spain), J. Hydrol., 420421, 301-318, 2012.

Bardsley, J., Solonen, A., Parker, A., Haario, H., and Howard, M.: An Ensemble Kalman Filter Using the Conjugate Gradient Sampler, Int. J. Uncert. Quant., 3, 357-370, doi:10.1615/Int.J.UncertaintyQuantification.2012003889, 2013.

Bates, P. and Anderson, M.: A two-dimensional finite-element model for river flow inundation, P. Roy. Soc. Lond. A. Mat., 440, 481-491, doi:10.1098/rspa.1993.0029, 1993.

Bellos, C., Soulis, J., and Sakkas, J.: Computation of twodimensional dam-break induced flows, Adv. Water Resour., 14, 31-41, 1991.

Casulli, V.: A Semi-implicit finite difference method for non hydrostatic, free-surface flows, Int. J. Numer. Meth. Fl., 30, 425-440, 1999.

Casulli, V. and Cheng, R.: Semi-implicit finite difference methods for three-dimensional shallow water flow, Int. J. Numer. Meth. Fl., 15, 629-648, doi:10.1002/fld.1650150602, 1992.

Courtier, P. and Talagrand, O.: Variational assimilation of meteorological observations with the adjoint vorticity equation, Part 2: Numerical results, Q. J. Roy. Meteor. Soc., 113, 1329-1368, 1987.

Daley, R.: Atmospheric Data Analysis, Cambridge University Press, 1st Edn., 1991.

Drikakis, D. and Tsangaris, S.: On the solution of the compressible Navier-Stokes equations using improved flux vector splitting methods, Appl. Math. Model., 17, 282-297, 1993.
Erpicum, S., Dewals, B., Archambeau, P., and Pirotton, M.: Dam break flow computation based on an efficient flux vector splitting, J. Comput. Appl. Math., 234, 2143-2151, 2010.

Evensen, G.: Sequential data assimilation with a nonlinear quasigeostrophic model using Monte-Carlo methods to forecast error statistics, J. Geophys. Res., 99, 10143-10162, 1994.

Goldstein, S.: Modern developments in fluid mechanics, Oxford Univ. Press, 1938.

Heniche, M., Secretan, Y., Boudreau., P., and Leclerc, M.: Twodimensional finite volume model for dam-break simulation, Adv. Water Resour., 23, 359-372, 2000.

Kalman, R.: A new approach to linear filtering and prediction problems. Transaction of the ASME, J. Basic Eng.-T. ASME, 82, 3545, 1960

Le Dimet, F.-X. and Talagrand, O.: Variational algorithms for analysis and assimilation of meteorological observations: theoretical aspects, Tellus, 38A, 97-110, 1986.

Lewis, J. M. and Derber, J. C.: The use of adjoint equations to solve a variational adjustment problem with advective constraints, Tellus, 37A, 309-322, 1985.

Lorenc, A.: Analysis methods for numerical weather prediction, Q. J. Roy. Meteor. Soc., 112, 1177-1194, 1986.

Martin, N. and Gorelick, S. M.: MODFreeSurf2D: A MATLAB surface fluid flow model for rivers and streams, Comput. Geosci., 31, 926-946, 2005.

Nocedal, J. and Wright, S.: Numerical Optimization, chap. LimitedMemory BFGS, Springer-Verlag, New York, 224-227, 1999.

Simon, D.: Optimal state estimation, Kalman, $H_{\infty}$, and nonlinear approaches, Wiley-Interscience, Hoboken, 2006.

Solonen, A., Haario, H., Hakkarainen, J., Auvinen, H., Amour, I., and Kauranne, T.: Variational ensemble Kalman filtering using limited memory BFGS, Electron T. Numer. Ana., 39, 271-285, 2012.

Toro, E. and Vazquez-Cendon, M.: Flux splitting schemes for the Euler equations, Comput. Fluids, 70, 1-12, 2012.

Ying, X., Jorgeson, J., and Wang, S.: Modeling Dam-Break flows using Finite Volume method on unstructured grid, Eng. Appl. Comput. Fluid Mech., 3, 184-194, 2009.

Yoon, T. and Kang, S.: Finite volume model for two-dimensional shallow water flows on unstructured grids, J. Hydraul. Eng.ASCE, 130, 678-688, 2004.

Zhang, M. and Wu, W.: A two dimensional hydrodynamic and sediment transport model for dam break based on finite volume method with quadtree grid, Appl. Ocean Res., 33, 297-308, 2011.

Zupanski, M.: Maximum Likelihood Ensemble Filter: Theoretical Aspects, Mon. Weather Rev., 133, 1710-1726, 2004. 